\title{
Conocimiento común del contenido del estudiante para profesor sobre fracciones y decimales
}

\section{Pre-service Teacher's Common Content Knowledge about the Fractions and Decimals}

\author{
Juan Francisco González Retana ${ }^{1}$ \\ Daniel Eudave Muñoz ${ }^{2}$
}

\begin{abstract}
Resumen. El aprendizaje de las fracciones y los decimales representa una dificultad para los estudiantes de educación primaria, lo que implica un reto para los profesores, en especial para quienes se están formando como docentes de educación básica. El presente artículo analiza el Conocimiento Común del Contenido sobre fracciones y decimales de estudiantes que aspiran a ser profesores de primaria. Se utilizó el Modelo del Conocimiento Matemático para la Enseñanza para analizar el conocimiento de los futuros maestros. Se aplicó una prueba de conocimientos sobre fracciones y decimales que se enseñan en la escuela primaria a 275 alumnos de $5^{\circ}$ y 7 으 semestres de dos escuelas formadoras de docentes. Entre los principales resultados se destaca que, en su mayoría, los futuros profesores tienen los conocimientos deseados en un egresado de la educación primaria y presentan mayor dificultad en resolver problemas que involucran el uso de fracciones que aquellos que implican decimales. Se detectaron algunos errores en los procesos de resolución de problemas que son elementales en el manejo de fracciones y decimales. El
\end{abstract}

Fecha de recepción: 15 de octubre de 2017. Fecha de aceptación: 13 de abril de 2018.

${ }^{1}$ Universidad Autónoma de Aguascalientes (México). bebe_jf@hotmail.com

2 Universidad Autónoma de Aguascalientes (México). deudave@correo.uaa.mx 
estudio aporta una reflexión sobre la implicación de estos conocimientos para las exigencias que el programa curricular de educación primaria demanda.

Palabras clave: conocimiento matemático para la enseñanza; conocimiento matemático del profesor; enseñanza de las matemáticas; formación de profesores.

\begin{abstract}
The learning of fractions and decimals represents a difficulty for elementary school students, which implies a challenge for teachers, and especially for those who are being trained as teachers of elementary school. This article analyzes the Common Knowledge Content about fractions and decimals of students who pursue to become elementary school teachers. The Model of Mathematical Knowledge for Teaching was used to analyze the future teachers' knowledge. A knowledge test about fractions and decimals taught in elementary school was applied to 275 students from 5th and 7th semesters at two teachers' training schools. Within the main findings, it is notable that most of the future teachers own the expected knowledge of an elementary school graduate; moreover, they have a higher difficulty to solve problems that involve the use of fractions than those involving decimals. Some errors were detected in the problem-solving processes which are basic when handling fractions and decimals. The study provides a reflection about the implication of this knowledge to address the demands of the elementary school curriculum program.
\end{abstract}

Keywords: mathematical knowledge for teaching; teacher's mathematical knowledge; teaching Mathematics; teachers' training.

\title{
1. INTRODUCCIÓN
}

Después de la asignatura de español, matemáticas ocupa el mayor número de horas para su estudio en la educación primaria en México. De las 900 horas anuales que se tienen programadas, 200 están destinadas al estudio de las matemáticas (Secretaría de Educación Pública [SEP], 2011).

En los últimos años México lleva a cabo ejercicios de evaluación a gran escala de los aprendizajes de los alumnos. Los resultados obtenidos muestran, de manera general, que los estudiantes de educación primaria (entre 6 y 12 años) alcanzan niveles deficientes en el aprendizaje de las matemáticas. 
Los resultados de las pruebas aplicadas en el marco del Plan Nacional para la Evaluación de los Aprendizajes (PLANEA) 2015 para sexto grado, indican que la mayoría de los alumnos se ubica en los niveles de logro 1 y 2 , donde solo escriben, comparan y resuelven problemas aritméticos con números naturales. Solamente unos pocos (cerca de 7\%) son capaces de resolver problemas que involucran números naturales, decimales y fraccionarios; o en donde el cálculo de promedios y medianas, así como la comparación de razones se ven involucrados (Instituto Nacional para la Evaluación de la Educación [INEE], 2015).

En el ámbito internacional, México también ha participado en evaluaciones a gran escala. En el Tercer Estudio Regional Comparativo y Explicativo (TERCE), aplicado en junio de 2013 a una muestra nacional de escuelas primarias, los resultados obtenidos mostraron que cerca de una cuarta parte (23\%) de los estudiantes alcanzó el nivel 1, esto es, pueden realizar tareas como "ordenar números naturales y decimales" (Flores y Díaz, 2016). 40\% se ubicó en el nivel 2 , donde son capaces de "resolver problemas simples con números naturales, decimales y fracciones" (Flores y Díaz, 2016: 21). 23\% obtuvo el nivel 3, algunas tareas que pueden realizar son: resolver problemas que contengan proporciones que involucran tanto las fracciones como los decimales (Flores y Díaz, 2016). Solo 14\% consiguió el nivel 4; además de efectuar las tareas de los niveles anteriores, son "capaces de interpretar datos de tablas o gráficas complejas para resolver problemas que incluyen conversión de unidades de medida y uso de números decimales y fracciones" (Flores y Díaz, 2016: 22).

Los Exámenes de Calidad y Logro Educativo (Excale) aportan información acerca de la dificultad que, para los alumnos de educación primaria, representan las fracciones y los decimales. En 2009, por ejemplo, en la aplicación en 6 o grado un tercio de los estudiantes (32\%) respondió correctamente reactivos relacionados con operaciones de fracciones, con denominadores distintos o sumar dos fracciones con diferente denominador. Solamente 18\% pudo ordenar en forma ascendente números decimales hasta milésimos, o resolver problemas de fracciones que relacionan dos números que representan la parte y el todo (INEE, 2012).

Los resultados anteriores son ejemplo de que, si bien a la asignatura de matemáticas se le otorga un lugar importante en el currículo de educación primaria, los alumnos no alcanzan los aprendizajes esperados. Si se considera que una de las figuras importantes en el aprendizaje escolar la constituye el profesor, sus conocimientos al respecto -tanto matemáticos como didácticos- se vuelven significativos. De ellos depende, en gran medida, la manera en que promueven los 
aprendizajes. Al respecto Hill, Rowan, y Ball (2005) señalan que los profesores necesitan un tipo de conocimiento matemático que les permita proporcionar explicaciones a sus estudiantes, analizar sus respuestas y emplear diversos recursos para representar conceptos matemáticos.

Las fracciones y los decimales son temas en que los docentes y los estudiantes para profesores presentan dificultades, tanto en su comprensión como para la enseñanza. Diversas investigaciones (Ball, 1990; Contreras, Carrillo, Zakaryan, Muñoz-Catalán y Climent, 2012; Durmuş, 2005; Gairín, 2013; Montes et al., 2015; Newton, 2008) resaltan las carencias de los estudiantes para profesores en los conocimientos acerca de dichas temáticas.

Con frecuencia se da por hecho que quienes ingresan a una institución para formarse como profesores cuentan con suficientes conocimientos matemáticos y que solo requieren un entrenamiento en didáctica, cuando en verdad muchos tienen pocas habilidades matemáticas y una pobre comprensión de los conceptos matemáticos, en especial de las fracciones, e incluso pueden tener muchas de las limitadas y erróneas comprensiones de los alumnos de educación básica (Newton, 2008). La deficiente formación matemática con que ingresan muchos estudiantes a las instituciones formadoras de docentes es reportada en otros estudios, por ejemplo Montes et al. (2015), quienes reportan que la mayoría tuvo su último contacto con las matemáticas varios semestres, o incluso años atrás.

Gairín (2004) hace un recuento de las limitaciones de los estudiantes para maestros en cuanto a sus conocimientos sobre los números racionales positivos: menciona que otorgan a la fracción un significado casi exclusivo como relación parte-todo, asociado además a modelos físicos; la notación decimal se reconoce exclusivamente como significado numérico, sin asociación a cantidades de magnitud; la relación entre las notaciones fraccionaria y decimal se da exclusivamente mediante procesos algorítmicos; solo se utilizan técnicas de cálculo para establecer relaciones de orden entre facciones, sin recurrir a modelos; solamente una tercera parte de los estudiantes considerados en este estudio establece relaciones de orden entre números decimales aplicando el principio del valor posicional; no admiten la densidad respecto al orden de los números racionales.

En general, las deficiencias que los profesores tienen se centran en: a) Otorgar a las fracciones un significado casi exclusivo como relación parte-todo; b) Un manejo de los números decimales como si fueran números enteros (Ávila y García, 2008; Gairín, 2004). 
Consideramos que los conocimientos matemáticos que los profesores adquieren antes de incorporarse al servicio docente resultan indispensables para el desarrollo de los aprendizajes de los estudiantes de primaria. Se espera que durante sus estudios los futuros profesores consoliden sus conocimientos matemáticos y adquieran los didácticos "que les permitan diseñar y aplicar estrategias didácticas eficientes para que los alumnos de educación primaria se apropien de las nociones, conceptos y procedimientos" (SEP, 2013: 5).

Coincidimos con Contreras, Carrillo, Zakaryan, Muñoz-Catalán y Climent (2012: 453) en que "el conocimiento especializado, que formará parte de su formación como maestros, necesita de un fuerte conocimiento matemático común previo, por ello no podemos obviar esta cuestión; al contrario, debemos tomarla como punto de partida". Bajo este supuesto, en este trabajo analizamos el Conocimiento Común del Contenido acerca de las fracciones y decimales de estudiantes que aspiran a ser profesores de primaria. Nos centramos en estos números, entre otras razones, a causa de los resultados que obtienen los alumnos de primaria en las distintas evaluaciones, por el valor que este tipo de números tienen en sí mismos y debido a sus aplicaciones en otros contenidos matemáticos pero, sobre todo, por la importancia que en esto tiene la participación del profesor. Para ello empleamos el Modelo del Conocimiento Matemático para la Enseñanza (MKT, por sus siglas en inglés) desarrollado por Ball $(2000,2003)$.

\section{FUNDAMENTOS TEÓRICOS}

\section{Modelo del Conocimiento Matemático para la EnSEÑAnZa (MKT)}

El interés por el análisis del conocimiento del profesor tomó fuerza a partir de los aportes de Shulman $(1986,1987)$. En sus trabajos se reconoce la importancia de los conocimientos para la enseñanza que necesitan los docentes. Se sostiene que se trata de un conjunto de conocimientos, amalgamados entre sí, acerca de la materia que se enseña y de su didáctica. Con relación al conocimiento de los profesores de matemáticas se han conformado diversas propuestas que pretenden delimitarlo y analizarlo, por ejemplo Ball (2003); Carrillo, Climent, Contreras y Muñoz-Catalán (2013); Godino, Batanero y Font (2007); Pino-Fan y Godino (2015); Schoenfeld y Kilpatrick (2008).

El Modelo del Conocimiento Matemático para la Enseñanza (MKT) es producto del trabajo desarrollado por Deborah Ball (Ball, 2000, 2003; Hill, Schilling 
y Ball, 2004; Hill, Rowan y Ball, 2005; Hill, Ball, y Schilling, 2008). Tiene origen en la necesidad de que los maestros desarrollen una práctica de enseñanza de calidad en matemáticas. Lo que interesa no es qué tanto de matemáticas sabe el maestro, sino cómo aprovecha ese conocimiento para la enseñanza. El Conocimiento Matemático para la Enseñanza es el conocimiento matemático, las habilidades y hábitos mentales que son empleados para el trabajo de enseñanza. En otras palabras, es el conocimiento matemático utilizado para llevar a cabo el trabajo de enseñanza de las matemáticas. El trabajo de enseñanza comprende todas aquellas tareas y responsabilidades que tienen los profesores para enseñar las matemáticas, tanto dentro como fuera del aula (Ball et al., 2006).

Ejemplos de este 'trabajo de enseñanza' incluyen explicar términos y conceptos a los estudiantes, interpretar las declaraciones y soluciones de los estudiantes, juzgar y corregir tratamientos de libros de texto de temas particulares, usar representaciones con precisión en el aula y proporcionar a los estudiantes ejemplos de conceptos matemáticos (Hill, Rowan y Ball, 2005: 373).

Si bien el MKT presenta algunas dificultades en cuanto a la definición de los diferentes subdominios (Montes et al., 2015), -por ejemplo, decidir cuándo termina el Conocimiento Común del Contenido y en qué momento comienza el Conocimiento Especializado del Contenido, lo mismo que demarcar los límites entre este y el Conocimiento en el Horizonte Matemático-, sigue siendo un modelo de análisis del conocimiento de los maestros, y un referente para el diseño de programas de formación del profesorado. De igual forma, ha dado pie a otros modelos como el de Carrillo et al. (2013).

El MKT se compone de: Conocimiento del Contenido Matemático y el Conocimiento Pedagógico del Contenido Matemático (Ball, 2005), cada uno conformado por tipos de conocimiento distintos, denominados subdominios (Figura 1). 


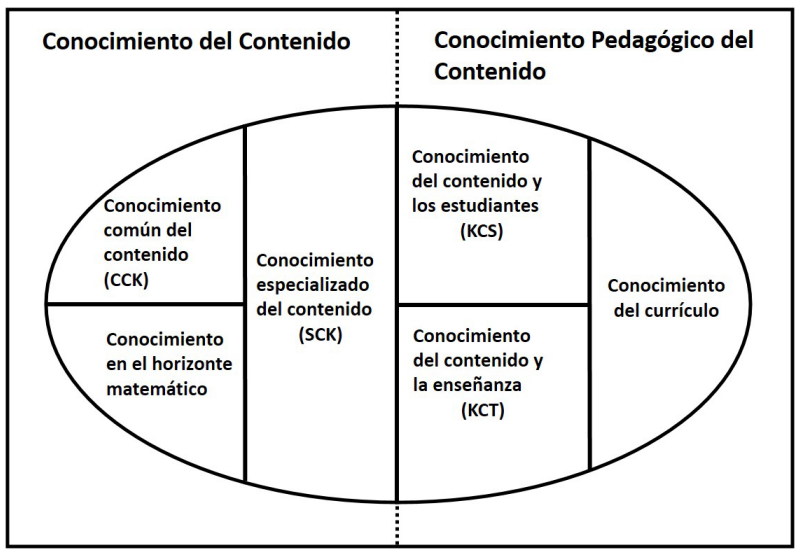

Figura 1. Modelo del Conocimiento Matemático para la Enseñanza (MKT) Tomado de (Hill, Ball y Schilling, 2008: 377).

El Conocimiento del Contenido se refiere a los conocimientos matemáticos que se supone posee una persona dedicada a la enseñanza de las matemáticas, como producto de su paso por la escuela básica y de su formación en docencia. Se conforma por tres subdominios:

- Conocimiento Común del Contenido (Common Content Knowledge, o CCK por sus siglas en inglés).

- Conocimiento Especializado del Contenido (Specialized Content Knowledge, o SCK por sus siglas en inglés).

- Conocimiento en el Horizonte Matemático (Ball, Themps y Phelps, 2008).

El Conocimiento Común de Contenido (CCK) está integrado por los conocimientos y las habilidades para resolver los problemas y ejercicios que se proponen en el nivel educativo donde el profesor se desempeña. Se trata de una serie de conocimientos que son empleados no solo en la enseñanza de las matemáticas sino en una variedad de entornos (Hill, Ball y Schilling, 2008), es decir, un conocimiento matemático que pueden tener otras personas que no son profesores y que no se decidan a enseñar. El Conocimiento Común del Contenido "es el relativo a la matemática teórico-práctica como cuerpo de conocimiento de posible aplicación a otros campos; puede considerarse similar a las matemáticas que se hallan en los libros de texto" (Montes et al., 2015: 40). Ball, Thames y 
Phelps (2008) señalan que los docentes necesitan un conocimiento matemático que les permita resolver los problemas que plantean a sus estudiantes.

El Conocimiento Especializado del Contenido (SKC) se compone del conocimiento y las habilidades de los profesores para el desarrollo de su trabajo en el área de matemáticas. Normalmente no se necesita para fines distintos de la enseñanza. Es un tipo de conocimiento matemático que los maestros deben poseer porque la enseñanza implica hacer que las características de un contenido particular sean visibles y aprendidas por los estudiantes.

El SKC implica el conocimiento de las razones por las que funcionan los procedimientos matemáticos (Hill, Ball y Schilling 2008). Involucra conocer la naturaleza matemática de los errores que cometen los alumnos y razonar si alguna de las soluciones que dan podría funcionar o no (Sosa, 2011). Ball, Thames y Phelps (2008) son enfáticos al señalar que se trata de un conocimiento matemático empleado en contextos de enseñanza de las matemáticas y que no es usado ni necesario, e incluso deseable, para cualquier otra persona que no se dedique a ello. ${ }^{3}$ EI SKC es, a decir de Montes et al. (2015), una de las grandes aportaciones de Ball y colaboradores, pues enfatiza los conocimientos que realmente requiere un profesor de matemáticas, a diferencia de otros usuarios de la matemática.

El Conocimiento en el Horizonte Matemático hace referencia al conocimiento para poder establecer las relaciones entre contenidos matemáticos de diferente complejidad y dificultad: lo que se conoce como una relación vertical (como puede ser la relación entre la aritmética con el álgebra) "un maestro de primaria tiene que saber con qué dificultades se enfrentarán sus estudiantes para sentar una base conceptual firme" (Fernández y Figueiras 2010: 294). O a las relaciones de los contenidos matemáticos, o de otra asignatura de un mismo nivel o grado educativo, entendidas como relaciones horizontales (las relaciones entre diferentes conceptos y de los conceptos de diferentes áreas de la matemática, como las que existen entre la aritmética y la geometría). Fernández y Figueiras (2010) mencionan que se trata de un conocimiento por medio del cual el profesor es consciente de la importancia de las relaciones entre los contenidos matemáticos del currículo. Este conocimiento va más allá del conocimiento del currículo; implica "una visión global de la educación matemática de los estudiantes" (Martínez, Giné, Fernández, Figueiras y Deulofeu 2011: 430). En otras palabras, el

\footnotetext{
3 Una discusión más amplia respecto a la definición de este subdominio la realizan (Flores-Medrano, Escudero Ávila, \& Carrillo, 2013).
} 
Conocimiento en el Horizonte Matemático involucra contar con un panorama longitudinal de los conceptos matemáticos y la relación que existe entre ellos. También "incluye las habilidades que tienen los profesores para saber la importancia que tiene un determinado contenido matemático durante su trayectoria curricular" (Sosa, 2011: 31).

El Conocimiento Pedagógico del Contenido (PCK) se concibe como los conocimientos indispensables para el desarrollo de los procesos de enseñanza y aprendizaje de las matemáticas. Está integrado por los siguientes subdominios:

a) Conocimiento del Contenido y los Estudiantes (KCS, por sus siglas en inglés).

b) Conocimiento del Contenido y la Enseñanza (KCT, por sus siglas en inglés).

c) Conocimiento del Currículo.

El Conocimiento del Contenido y los Estudiantes (KCS) comprende conocer a los alumnos y lo que saben de matemáticas, entrelaza un conocimiento del contenido con el conocimiento de cómo los estudiantes piensan y aprenden (Hill, Ball y Schilling, 2008). Implica que el profesor sea capaz de anticipar lo que los alumnos pueden pensar y lo que encontrarán confuso (Ball, Thames y Phelps, 2008). Al asignar una tarea, los maestros necesitan anticipar lo que los estudiantes probablemente harán con ella, y si les resultará fácil o difícil. Los docentes necesitan predecir lo que los alumnos encontrarán interesante y motivador. Este conocimiento ayuda al profesor a diseñar actividades para abordar contenidos matemáticos.

Los maestros también deben ser capaces de escuchar e interpretar el pensamiento emergente e incompleto de los estudiantes, expresado en la manera en que los alumnos usan el lenguaje matemático. Cada una de estas tareas requiere de una interacción entre la comprensión matemática específica y la familiaridad con los estudiantes y su pensamiento matemático (Hill, Ball y SchiIling, 2008). Al respecto Sosa (2011: 32) señala que los profesores "hacen una imagen de lo que posiblemente harán los alumnos en las tareas matemáticas que les asignan [dentro de este subdominio] se considera la capacidad [de] los maestros para escuchar y comprender el pensamiento de los alumnos en su lenguaje usual".

El Conocimiento del Contenido y la Enseñanza $(K C T)$ involucra conocer diversas maneras de acercar algún contenido matemático a los alumnos, esto es, ventajas educativas de utilizar o seguir una determinada estrategia didáctica 
para enseñar un contenido matemático concreto. El profesor decide con qué ejemplos empezar un tema, cuáles utilizar para adentrarlos más en el contenido, "qué aportaciones de los alumnos tomar en cuenta, cuáles ignorar y cuáles destacar para usarlas posteriormente... cuándo aclarar más una idea, cuándo hacer una nueva pregunta o encomendar una nueva tarea para fomentar más el pensamiento matemático de los alumnos" (Ball, Thames y Phelps 2008: 401). Los maestros valoran las ventajas y desventajas de ciertos diseños de instrucción para identificar aquellos que les pueden ser de utilidad para acercar a los estudiantes a los aprendizajes matemáticos.

El Conocimiento del Currículo supone el conocimiento de la composición y estructura curricular. El profesor tiene el conocimiento de la manera en que está organizado el currículo del nivel y el grado educativo donde se desempeña, lo cual le permite la planificación de las actividades para el desarrollo del pensamiento matemático en los estudiantes (Ball, Thames y Phelps 2008).

En este texto nos centramos en el análisis del subdominio Conocimiento Común del Contenido, de manera particular, en los conocimientos sobre fracciones y decimales de los estudiantes para profesores de educación primaria. Consideramos que un maestro de educación primaria debe contar con un conocimiento acerca del uso y manejo de fracciones y decimales, que le permitan resolver problemas o ejercicios planteados en el currículo de educación primaria (SEP, 2011). Entre los principales conocimientos con que debe contar un profesor de educación primaria -según los planes, programas de estudio y libros de texto de la misma-, distinguimos los siguientes:

- Leer y escribir números decimales.

- Leer y escribir números decimales empleando expresiones finitas e infinitas.

- Trasformar números decimales a fracciones decimales o fracciones comunes.

- Comparar y ordenar fracciones y números decimales.

- Comprender la propiedad de densidad de los números decimales y las fracciones.

- Resolver problemas con números decimales y fracciones que impliquen:

- Adición

- Sustracción

- Multiplicación

- División 
- Conocer las propiedades de las operaciones con números decimales y las relaciones entre ellas.

- Ubicar fracciones y números decimales en la recta numérica.

- Leer y escribir fracciones:

- Propias, impropias y mixtas

- Equivalentes

- Decimales

- Resolver problemas que impliquen el uso de fracciones equivalentes.

- Simplificar, a su mínima expresión, fracciones propias, impropias y mixtas.

- Resolver problemas con fracciones comunes, impropias y mixtas, que implican:

- Adición

- Sustracción

- Multiplicación

- División

- Resolver problemas que implican el cálculo de una fracción de un número natural.

Con lo anterior no pretendemos agotar el núcleo de conocimientos sobre fracciones y decimales que debe poseer un futuro profesor. Al contrario, consideramos que los enlistados implican otro tipo de conocimientos no explícitos en los programas de educación primaria y que son necesarios para un adecuado desempeño docente; por ejemplo, la comprensión de que tanto las fracciones como los decimales pertenecen al conjunto de los números racionales, lo cual involucra un conocimiento mucho más amplio que solamente la capacidad de operar con ellos. Otro elemento importante radica en la comprensión de las diversas interpretaciones de las fracciones: parte-todo, cociente, medida, razón y operador (Kieren, 1988). Lo mismo ocurre con los números decimales, pues su conocimiento demanda al futuro profesor entender que son aquellos que pueden representarse en forma de fracción decimal (Ávila y García, 2008).

${ }^{4}$ Las fracciones decimales son las que pueden expresarse con un numerador entero y un denominador que es una potencia de diez, por ejemplo $\frac{3}{10}$ y $\frac{1}{100}$ son fracciones decimales; también son ejemplos de fracciones decimales $\frac{1}{2}$ y $\frac{3}{5^{\prime}}$, ya que se pueden encontrar fracciones equivalentes a un medio y a tres quintos cuyos denominadores sean una potencia de 10 . 


\section{METODOLOGÍA DE LA INVESTIGACIÓN}

Se trata de una investigación cuantitativa en donde empleamos el método de la encuesta. Como instrumento de obtención de información se diseñó y administró un examen de conocimientos sobre fracciones y decimales enseñados en la escuela primaria, compuesto por 30 reactivos. La Tabla 1 muestra el propósito de cada reactivo de la prueba.

Tabla 1. Propósitos por reactivo de la prueba de conocimientos sobre fracciones y decimales. ${ }^{5}$

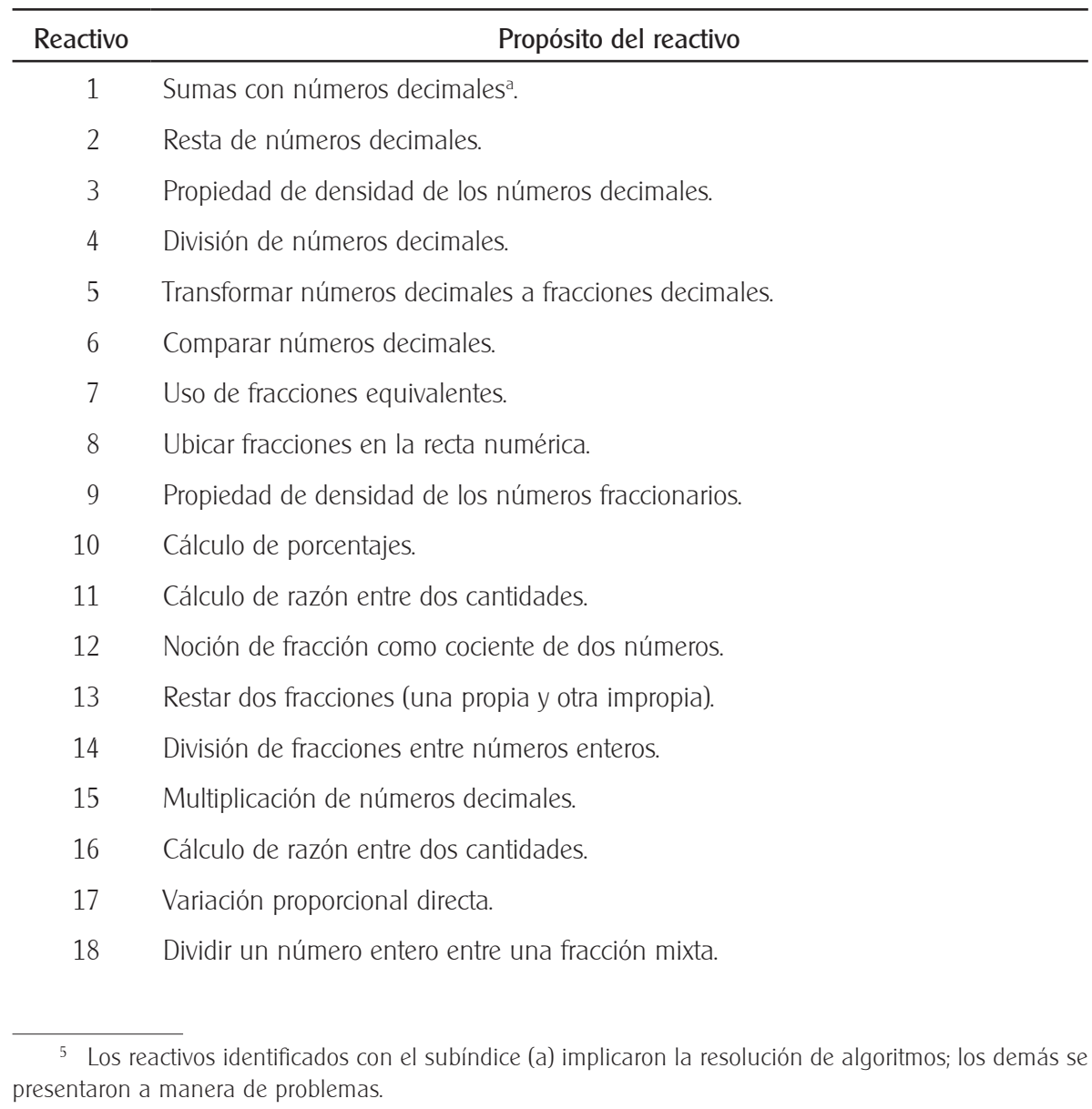




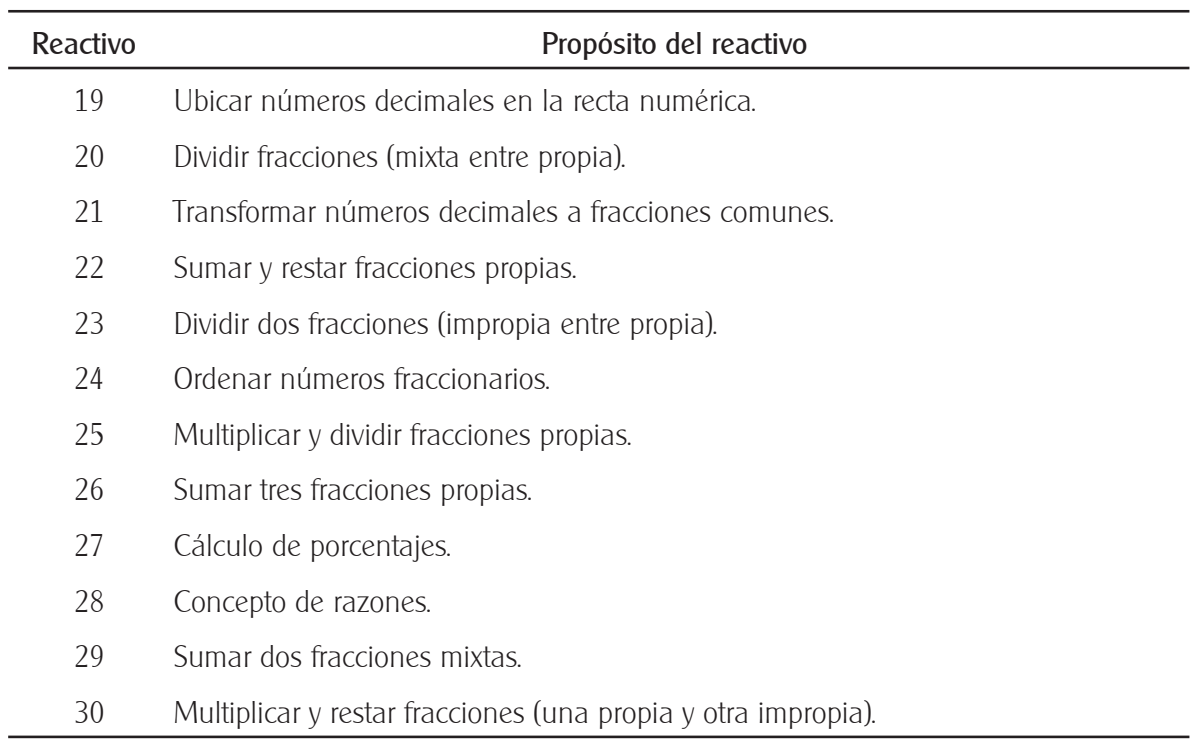

Un total de 275 estudiantes para profesores de la Licenciatura en Educación Primaria (LEP) de dos Escuelas Normales del estado de Durango, en México, respondieron la prueba. 96 (62 de $5^{\circ}$ semestre y 34 de 7으) de una Normal urbana y 179 (84 de 5으 y 95 de 7으) de una Normal rural.

Para la validación de los reactivos que conformaron la prueba se emplearon los supuestos de la Teoría Clásica de los Test (TCT). Una de las ideas que la TCT sostiene es que, para validar una prueba, es necesario calcular el Índice de Confiabilidad y el Error de Medida, además de estimar el Índice de Discriminación y el Índice de Dificultad de cada reactivo. Con base en los resultados que obtuvimos en una aplicación piloto, ajustamos algunos reactivos y eliminamos otros. Con los resultados de los análisis anteriores concluimos que la prueba resultaba confiable, aunque moderadamente fácil de resolver para los estudiantes para profesores. El cálculo del Índice de Confiabilidad se elaboró mediante el coeficiente del alfa de Cronbach, obteniendo un valor de 0.808. El error de medida fue de $0.192 .^{6}$

6 El error de medida puede tener valores de 0 a 1 ; mientras más se acerca a 0 se dice que el error tiende a minimizarse, por lo cual la prueba puede resultar adecuada para los fines que fue diseñada (Abad et al., 2004). 


\section{RESULTADOS}

Para el análisis, las respuestas fueron codificadas de la siguiente manera: 0 (cero) para una respuesta incorrecta y 1 para una respuesta correcta. Dado que el total de reactivos fue de 30 , un puntaje para quien respondió correctamente todos los ítems fue de 30 aciertos; para el que respondió de forma incorrecta todo el examen, su puntaje sería de 0 (cero).

El promedio de aciertos para el total de la muestra fue $24.1(s=4.2)_{1}^{7}$ el cual es muy similar al reportado por Newton (2008) (con un promedio de 23.25 de un total de 30 reactivos, para las habilidades de cálculo, en un examen previo a un curso de matemáticas contestado por estudiantes para profesores, y a un promedio de 27.6 posterior al citado curso). El puntaje de aciertos más alto fue de 30 y el menor de 10 .

El promedio (24.1) sugiere que, en general, los participantes respondieron correctamente casi todos los reactivos. Lo que nos lleva a considerar, de entrada, que los estudiantes para profesores de las escuelas en donde realizamos el estudio cuentan con conocimientos que les permiten resolver problemas y algoritmos que involucran fracciones y decimales estudiados en la escuela primaria (Ball, Thames y Phelps, 2008).

Conviene matizar el hallazgo anterior porque 10 estudiantes obtuvieron 15 o menos respuestas correctas, lo cual llama la atención, pues el examen fue elaborado considerando contenidos matemáticos que se estudian en la escuela primaria (ver Tabla 1). De estos 10, cuatro cursaban $7^{\circ}$ semestre de la LEP y en breve serían profesores en servicio, con un grupo de alumnos de primaria a su cargo. ${ }^{8}$

Se calculó el promedio de aciertos por escuela, semestre y grupo. Los estudiantes de la Normal urbana obtuvieron un promedio de $24.6(s=3.9)$ aciertos, mientras que los de la Normal rural alcanzaron $23.8(s=4.3)$. Aun cuando la diferencia en los promedios fue menor a un punto, se consideró pertinente analizar si resultaba estadísticamente significativa. Dado que los datos no presentaron una distribución normal ${ }^{9}$ empleamos la prueba $U$ de Mann-Whitney, ${ }^{10}$ mediante la cual se encontró que entre los resultados globales de ambas

\footnotetext{
${ }^{7}$ El valor $s$ corresponde a la desviación estándar o desviación típica.

8 El Plan de Estudios de la Licenciatura en Educación Primaria 2012 tiene una duración de 8 semestres.

${ }_{9}^{9}$ Para probar la normalidad de los datos se utilizó la prueba de Komogorov-Smirnov, que permite comparar la distribución de un conjunto de datos de acuerdo con una distribución teórica específica (normal).

${ }^{10}$ La prueba U de Mann-Whitney se sugiere cuando la distribución de los datos comparados no presenta una distribución normal.
} 
Escuelas Normales no existen diferencias estadísticamente significativas (esto es, sin hacer distinción entre semestres y grupos).

Realizamos una comparación entre el promedio de aciertos por escuela y semestre que cursaban los estudiantes. Lo anterior porque nos resulta útil distinguir los conocimientos entre alumnos de diferentes semestres y con base en el supuesto de que los conocimientos de los alumnos de $7^{\circ}$ eran distintos a los de $5^{\circ}$, no solo por los dos semestres de distancia sino también debido a la experiencia al frente de un grupo de alumnos de primaria que proporcionan las jornadas de práctica intensiva que realizan los estudiantes de $7^{\circ}$, además de que este análisis contribuye a una descripción más detallada de los conocimientos sobre fracciones y decimales de los futuros profesores. En la Figura 2 aparece esta comparación.

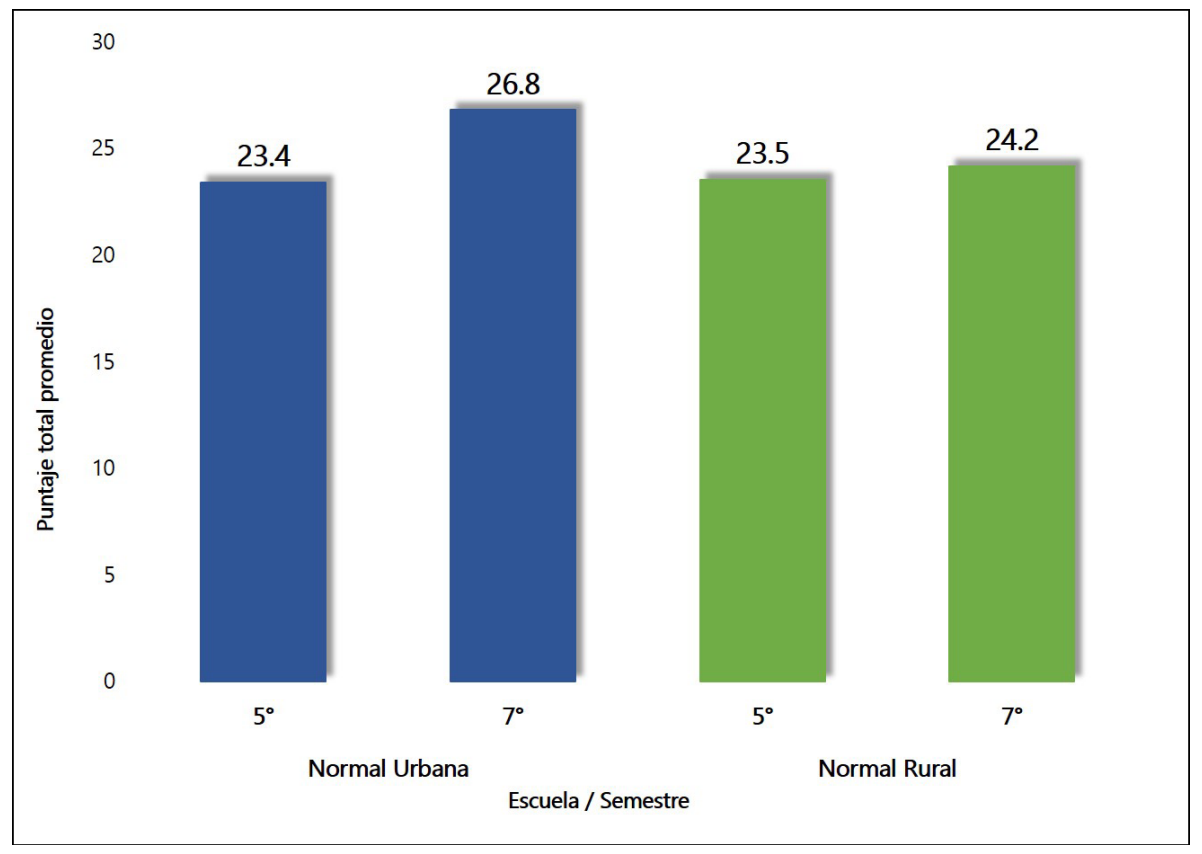

Figura 2. Puntajes promedio del examen de conocimientos matemáticos por semestre en cada escuela normal. 
Considerando que todos los alumnos ya cursaron las asignaturas de la licenciatura que se relacionan con la enseñanza de las matemáticas, ${ }^{11}$ se esperarían resultados similares en todos los semestres. Sin embargo, las diferencias nos plantean situaciones interesantes. La diferencia más grande de los promedios entre semestres es cercana a los 3 puntos y medio (de $5^{\circ}$ a $7^{\circ}$ semestres de la Normal urbana). Llama la atención el hecho de que la diferencia entre los semestres de la Normal rural no sea ni de un dígito.

Con el fin de observar si las diferencias eran estadísticamente significativas, elaboramos pruebas de contraste de hipótesis. La Tabla 2 muestra un resumen de los datos contrastados, la prueba empleada ${ }^{12}$ y su resultado. Tenemos que en las comparaciones donde intervienen los resultados de los alumnos de $7^{\circ}$ semestre de la Normal urbana resultan estadísticamente significativas. Parece entonces que dichas diferencias no se deben al azar.

Tabla 2. Comparación de diferencias entre los puntajes del examen de conocimientos matemáticos por escuela y semestre.

\begin{tabular}{ll}
\hline \multicolumn{1}{c}{ Relación } & \multicolumn{1}{c}{ Conclusión } \\
\hline $5^{\circ}-7^{\circ}$ Normal Urbana & Diferencia estadísticamente significativa (3.4 puntos). \\
$5^{\circ}-7^{\circ}$ Normal Rural & $\begin{array}{l}\text { No existe diferencia estadísticamente significativa (menos de un } \\
\text { punto). }\end{array}$ \\
$5^{\circ}$ Urbana $-5^{\circ}$ Rural & No existe diferencia estadísticamente significativa. \\
$7^{\circ}$ Urbana $-7^{\circ}$ Rural & Diferencia estadísticamente significativa (2.6 puntos). \\
\hline
\end{tabular}

Estos resultados sugieren diversas explicaciones, por ejemplo, pueden deberse a los conocimientos que los estudiantes de $7^{\circ}$ semestre de la Normal urbana hubieran adquirido durante su paso por la educación básica y media superior (por tener mejores profesores o mejores condiciones de aprendizaje). Otra posible explicación es que las diferencias en realidad se deban a los conocimientos que desarrollaron durante los cursos de matemáticas que estudiaron como parte de su formación docente, donde quizá lograron consolidar los

\footnotetext{
${ }^{11}$ Los cursos considerados son: Aritmética: su aprendizaje y enseñanza; Álgebra: su aprendizaje y enseñanza; Geometría: su aprendizaje y enseñanza, y Procesamiento de la Información Estadística.

12 En todos los casos empleamos el estadístico U de Mann-Whitney, dado que la distribución de los datos no presentó normalidad. Las pruebas de normalidad se elaboraron por medio de los estadísticos de Kolmogorov-Smirnov y Shapiro-Wilk.
} 
conocimientos acerca de las fracciones y decimales que se consideraron en el examen (debido tal vez, también a mejores profesores y estrategias de enseñanza) o, lo que es lo mismo, un desarrollo en el conocimiento común del contenido matemático.

Se calculó el promedio de aciertos por grupo en cada semestre de ambas escuelas normales; la Tabla 3 contiene dicha información y en ella aparecen resaltados con doble asterisco $\left({ }^{* *}\right)$ los más bajos y señalados con un asterisco $\left({ }^{*}\right)$ los más altos. En la Normal rural la diferencia entre los grupos B y D de $7^{\circ}$ es de 5 aciertos. Se advierte que los resultados del grupo B de $7^{\circ}$ semestre de la Normal rural son similares a los obtenidos por el grupo de $7^{\circ}$ de la Normal urbana.

Tabla 3. Puntaje promedio por grupo.

\begin{tabular}{|c|c|c|c|c|c|c|}
\hline Escuela & Semestre & Grupo & $\begin{array}{l}\text { Puntaje } \\
\text { promedio }\end{array}$ & $\begin{array}{l}\text { Desviación } \\
\text { estándar }\end{array}$ & $\begin{array}{l}\text { Puntaje } \\
\text { mínimo }\end{array}$ & $\begin{array}{l}\text { Puntaje } \\
\text { máximo }\end{array}$ \\
\hline \multirow{3}{*}{ Normal Urbana } & \multirow{2}{*}{$5^{\circ}$} & A & 24.4 & 2.7 & 19 & 28 \\
\hline & & B & $22.2^{* *}$ & 4.9 & 14 & 29 \\
\hline & $7 ㅇ$ & U & 26.8 & 2.8 & 19 & 30 \\
\hline \multirow{8}{*}{ Normal Rural } & \multirow{4}{*}{$5^{\circ}$} & A & 23.5 & 5.8 & 10 & 30 \\
\hline & & B & $22.4^{* *}$ & 4.7 & 10 & 29 \\
\hline & & C & $22.4^{* *}$ & 4.2 & 14 & 29 \\
\hline & & $\mathrm{D}$ & $25.6^{*}$ & $2.1^{*}$ & 21 & 28 \\
\hline & \multirow{4}{*}{7 은 } & A & 24.2 & 3.4 & 15 & 29 \\
\hline & & B & $26.7^{*}$ & $3.6^{*}$ & 16 & 30 \\
\hline & & C & 23.6 & 3.9 & 14 & 29 \\
\hline & & $\mathrm{D}$ & $21.7^{* *}$ & 4.1 & 14 & 29 \\
\hline
\end{tabular}

Aunque en conjunto existe una diferencia estadísticamente significativa entre el promedio de aciertos de los alumnos del $7^{\circ}$ semestre de la Normal urbana y los de la Normal rural, al analizar el promedio de aciertos por grupo encontramos que el de $7^{\circ} \mathrm{B}$ de la Normal rural es muy similar al $7^{\circ} \mathrm{U}$ de la Normal urbana. Lo más notorio es la diferencia entre los resultados de una misma escuela, en este caso en el $7^{\circ}$ semestre de la Normal rural. 
Resalta también que todos los grupos de $5^{\circ}$ semestre de la Normal rural obtuvieron un promedio de aciertos por encima del $5^{\circ} \mathrm{B}$ de la Normal urbana. Incluso el $5^{\circ} \mathrm{D}$ de la Normal rural obtuvo un valor ligeramente mayor al $5^{\circ} \mathrm{A}$ de la Normal urbana. Estas diferencias entre grupos, independientemente del tipo de escuela e incluso el semestre, hacen suponer, como posible explicación, que las diferencias se deben al o los docentes que les impartieron los cursos relacionados con la formación matemática, o al menos el de "Aritmética: su aprendizaje y enseñanza", donde se estudian las fracciones y los decimales. Se puede inferir así, como lo sugieren Conteras et al. (2012), el potencial que puede tener la Escuela Normal para lograr mejores aprendizajes aritméticos de los futuros profesores, a pesar de su origen sociocultural y sus antecedentes académicos.

Hicimos un proceso de análisis más detallado al examinar el porcentaje de respuesta correcta para cada reactivo. Con lo anterior se pretendió ir configurando los conocimientos de los futuros profesores sobre fracciones y decimales. Para ello analizamos el porcentaje de respuesta correcta para cada reactivo. La Tabla 4 presenta cada uno de ellos y el conocimiento que se pretendía medir con cada reactivo. Los porcentajes de respuesta correcta más altos corresponden a los reactivos que involucraron números decimales, mientras que los más bajos fueron aquellos que implicaron el uso de fracciones. Este hecho quizá tenga una explicación en que los decimales tienen una ventaja sobre las fracciones, pues resulta más fácil operar con ellos, pues los algoritmos son los mismos que se emplean en los números naturales, solo que existe la dificultad al elegir el lugar en dónde se debe colocar el punto decimal en el resultado (Ávila y García, 2008). 
Tabla 4. Porcentaje de respuesta correcta por reactivo. ${ }^{13}$

\begin{tabular}{|c|c|c|}
\hline Reactivo & Especificación & $\%$ de respuesta correcta \\
\hline 6 & Comparar números decimales. & 98.9 \\
\hline 21 & Resolver problemas que implican transformar números decimales a fracciones comunes. & 93.3 \\
\hline 15 & Resolver problemas que implican multiplicación de números decimales. & 92.6 \\
\hline 19 & Resolver problemas que implican ubicar números decimales en la recta numérica. & 92.2 \\
\hline 3 & Resolver problemas que implican la propiedad de densidad de los números decimales. & 90.0 \\
\hline 17 & Resolver problemas que implican variación proporcional directa. & 89.6 \\
\hline 1 & Resolver sumas con números decimales. & 89.2 \\
\hline 12 & Resolver problemas que implican la noción de fracción como cociente de dos números. & 88.5 \\
\hline 4 & Resolver problemas que implican división de números decimales. & 87.7 \\
\hline 5 & Resolver problemas que implican transformar números decimales a fracciones decimales. & 86.6 \\
\hline 10 & Resolver problemas que implican el cálculo de porcentajes. & 86.2 \\
\hline 14 & Resolver problemas que implican división de fracciones entre números enteros. & 86.2 \\
\hline 11 & Resolver problemas que implican el cálculo de razón entre dos cantidades. & 85.9 \\
\hline 16 & Resolver problemas que implican el cálculo de razón entre dos cantidades. & 85.9 \\
\hline 24 & Resolver problemas que implican ordenar números fraccionarios. & 85.5 \\
\hline 2 & Resolver problemas que implican resta de números decimales. & 84.0 \\
\hline 28 & Resolver problemas que implican el concepto de razones. & 83.3 \\
\hline 20 & Resolver problemas que implican dividir fracciones (mixta entre propia). & 82.9 \\
\hline 13 & Resolver problemas que implican restar dos fracciones (una propia y otra impropia). & 82.5 \\
\hline 27 & Resolver problemas que implican el cálculo de porcentajes. & 82.2 \\
\hline 29 & Resolver problemas que implican sumar dos fracciones mixtas. & 80.3 \\
\hline 18 & Resolver problemas que implican dividir un número entero entre una fracción mixta. & $\mathbf{7 4 . 7}$ \\
\hline 7 & Resolver problemas que implican el uso de fracciones equivalentes. & 72.9 \\
\hline 8 & Resolver problemas que implican ubicar fracciones en la recta numérica. & 72.9 \\
\hline 26 & Resolver problemas que implican sumar tres fracciones propias. & 71.4 \\
\hline 9 & Resolver problemas que implican la propiedad de densidad de los números fraccionarios. & 68.8 \\
\hline 30 & Resolver problemas que implicar multiplicar y restar fracciones (una propia y otra impropia). & 61.0 \\
\hline 23 & Resolver problemas que implican dividir dos fracciones (impropia entre propia). & 58.4 \\
\hline 22 & Resolver problemas que implican sumar y restar fracciones propias. & 55.8 \\
\hline 25 & Resolver problemas que implican multiplicar y dividir fracciones propias. & 44.6 \\
\hline
\end{tabular}

Para analizar con mayor detalle las respuestas de los estudiantes, se organizaron los reactivos que conformaron el examen en grupos, de acuerdo con el conocimiento que implicaron para su solución. Formamos cinco conjuntos de reactivos que involucraron:

a) La realización de operaciones básicas (suma, resta multiplicación y división) con números decimales.

b) El manejo de algunas propiedades (densidad) de los números decimales.

c) El uso operaciones básicas (suma, resta multiplicación y división) con fracciones. ${ }^{14}$

${ }^{13}$ Los reactivos 10, 11, 28 y 27 corresponden al cálculo de razones y porcentajes. Se incluyeron en la prueba puesto que, al igual que los decimales y las fracciones, pertenecen al conjunto de los números racionales.

${ }^{14}$ Este conjunto de reactivos se dividió a su vez en dos grupos: aquellos que implicaban el uso de una operación y otros que involucraron dos o más. 
d) El manejo de algunas propiedades (equivalencia, densidad, entre otros) de las fracciones.

e) Operaciones básicas con números decimales y fracciones.

La Tabla 5 presenta los porcentajes de respuesta correcta para los reactivos que involucraron el uso de operaciones básicas (suma, resta, multiplicación y división) con números decimales. Se incluyeron cuatro reactivos (1, 2, 4 y 15), tres presentados como problemas y uno que implicó la resolución de un algoritmo de suma (1).

Tabla 5. Reactivos que implican operaciones básicas con números decimales.

\begin{tabular}{clc}
\hline Reactivo & \multicolumn{1}{c}{ Especificación } & $\begin{array}{c}\text { \% de respuesta } \\
\text { correcta }\end{array}$ \\
\hline 1 & Resolver sumas con números decimales. & 89.2 \\
2 & $\begin{array}{l}\text { Resolver problemas que implican resta de } \\
\text { números decimales. }\end{array}$ & 84.0 \\
& $\begin{array}{l}\text { Resolver problemas que implican división de } \\
\text { números decimales. }\end{array}$ & 87.7 \\
& $\begin{array}{l}\text { Resolver problemas que implican multiplicación } \\
\text { de números decimales. }\end{array}$ & 92.6 \\
\hline
\end{tabular}

Como se observa, la gran mayoría de los estudiantes respondió de manera correcta los reactivos de este grupo, lo cual nos lleva a suponer que los futuros profesores, en general, cuentan con el conocimiento para resolver problemas que implican el uso de suma, resta, multiplicación y división con números decimales. Es importante recordar que los reactivos hacen referencia a contenidos que se estudian en la escuela primaria.

Aunque los resultados anteriores nos hacen pensar que los estudiantes poseen los conocimientos básicos sobre fracciones y decimales, nos propusimos analizar el tipo de respuestas proporcionadas por estos futuros profesores. Observamos que quienes contestaron de manera incorrecta, por lo general cometían el mismo error. La Figura 3 muestra la forma en que uno de los participantes resolvió el reactivo 1. Como se aprecia, el acomodo de los sumandos que hace es erróneo. El error es colocar los números decimales en posición inadecuada, pues el número 6 del segundo sumando (12.6) que se ubica en la posición de los décimos, al resolver 
la suma, el alumno lo coloca en el lugar de los centésimos. Este error Centeno (1988) lo identifica como uno de los más comunes, y se relaciona con el concepto de número decimal. Además, resulta interesante que el porcentaje de alumnos que respondieron de manera incorrecta este reactivo (10.8\%) representa 29 estudiantes, la mayoría de ellos (20) de la Normal rural.

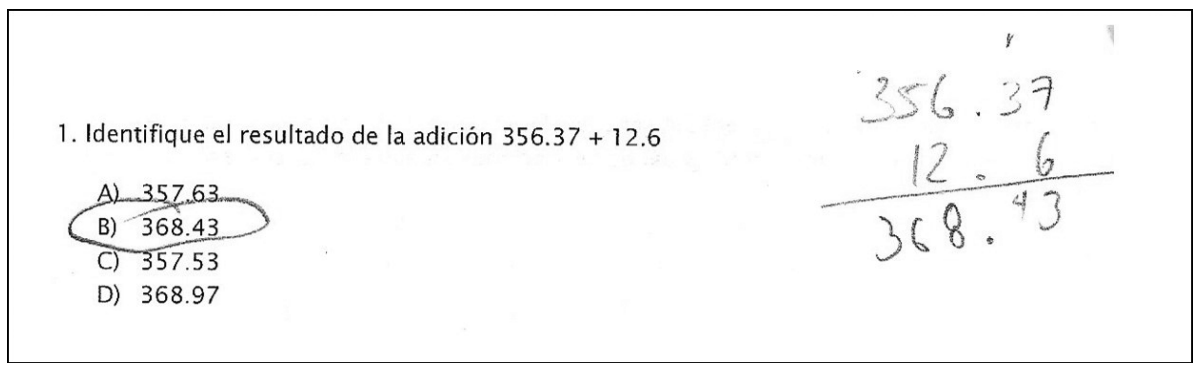

Figura 3. Procedimiento de solución de un estudiante al reactivo 1.

En la Tabla 6 se presenta el porcentaje de aciertos para los reactivos que implicaron el manejo de alguna propiedad de los decimales. El reactivo 5 registró el menor porcentaje de respuesta correcta. En dicho reactivo se solicitó transformar el número 8.05 a una fracción decimal (Figura 4).

Tabla 6. Reactivos que implican el manejo de propiedades de los números decimales.

\begin{tabular}{clc}
\hline Reactivo & \multicolumn{1}{c}{ Especificación } & $\begin{array}{c}\text { \% de respuesta } \\
\text { correcta }\end{array}$ \\
\hline 3 & $\begin{array}{l}\text { Resolver problemas que implican la propiedad de densidad } \\
\text { de los números decimales. }\end{array}$ & 90.0 \\
5 & $\begin{array}{l}\text { Resolver problemas que implican transformar números } \\
\text { decimales a fracciones decimales. }\end{array}$ & 86.6 \\
6 & $\begin{array}{l}\text { Comparar números decimales. } \\
19\end{array}$ & $\begin{array}{l}\text { Resolver problemas que implican ubicar números } \\
\text { decimales en la recta numérica. }\end{array}$ \\
\hline 21 & $\begin{array}{l}\text { Resolver problemas que implican transformar números } \\
\text { decimales a fracciones comunes. }\end{array}$ & 98.9 \\
\hline
\end{tabular}


Aunque casi todos los estudiantes (86.6\%) respondieron el reactivo 5 de manera correcta, el resto, 36 estudiantes (13.4\%), lo hizo incorrectamente. Estos alumnos, en su mayoría, seleccionaron la opción D como correcta. Encontramos respuestas como la que muestra la Figura 4 La solución del estudiante hace suponer que al trasformar el número 8.05 a una fracción decimal consideró la cantidad de cifras que lo componen (3) y, con base en ello, seleccionó un número con la misma cantidad de ceros (en este caso 1000). Sobresale su confusión respecto a la posición del numerador y del denominador.

5. En la clase de matemáticas el docente le solicita a Sofía que escriba en el pizarrón la fracción decimal equivalente al número 8.05 ¿Cuál de las opciones muestra la fracción solicitada por el docente?
A) $\frac{1000}{805}$
B) $\frac{805}{100}$
C) $\frac{100}{805}$
D) $\frac{805}{1000}$

Figura 4. Respuesta de un estudiante al reactivo 5.

En la Tabla 7 aparecen los porcentajes de respuesta correcta en los reactivos que involucraron la resolución de operaciones básicas con fracciones. Alrededor de ocho de cada 10 futuros profesores fue capaz de resolver problemas que implicaron operaciones básicas (suma, resta, multiplicación o división) con fracciones, lo anterior nos permite sostener que quienes participaron en este estudio poseen el conocimiento matemático que demandan situaciones como las planteadas en los reactivos. 
Tabla 7. Reactivos que implican operaciones básicas con fracciones.

\begin{tabular}{clc}
\hline Reactivo & \multicolumn{1}{c}{ Especificación } & $\begin{array}{c}\text { \% de respuesta } \\
\text { correcta }\end{array}$ \\
\hline 13 & $\begin{array}{l}\text { Resolver problemas que implican restar dos fracciones } \\
\text { (una propia y otra impropia). }\end{array}$ & 82.5 \\
20 & $\begin{array}{l}\text { Resolver problemas que implican dividir fracciones } \\
\text { (mixta entre propia) }\end{array}$ & 82.9 \\
23 & $\begin{array}{l}\text { Resolver problemas que implican dividir dos fracciones } \\
\text { (impropia entre propia). }\end{array}$ & 82.9 \\
26 & $\begin{array}{l}\text { Resolver problemas que implican sumar fracciones } \\
\text { propias (tres sumandos). }\end{array}$ & 71.4 \\
29 & $\begin{array}{l}\text { Resolver problemas que implican sumar dos } \\
\text { fracciones mixtas. }\end{array}$ & 80.3 \\
\hline
\end{tabular}

Al analizar las respuestas de los estudiantes con relación a este conjunto de preguntas centramos la atención en el reactivo 26, donde se esperaba que los estudiantes resolvieran un problema que implicaba la suma de tres fracciones propias con denominadores equivalentes. Cerca de una tercera parte de los futuros profesores (28\%, que representan 77 alumnos) no fue capaz hacerlo. Resulta interesante que la mayoría eran alumnos de la Normal rural (32 de $5^{\circ}$ semestre y $31 \mathrm{de} 7^{\circ}$ ). Al analizar las respuestas que respondieron incorrectamente se encontró que una cantidad importante $(12.3 \%$, es decir 33 estudiantes) siguió un procedimiento de resolución muy parecido al que presenta la Figura 5.

26. En la escuela de Carlos promocionaron la actividad "El kilómetro del libro". El objetivo era formar $1 \mathrm{~km}$ de libros alineados sobre el piso. Durante tres días sus compañeros lo construyeron: el primer día avanzaron $\frac{2}{3} \mathrm{de} / \mathrm{km}$; el segundo, $\frac{1}{6} \mathrm{~km}$; y el tercero, $\frac{1}{12}$ de $\mathrm{km}$. ¿Qué fracción representa la parte que les faltó para completar el kilómetro?
A) $\frac{1}{3}$
B) $\frac{1}{2}$
(C) $\frac{1}{10}$
D) $\frac{1}{12}$

Figura 5. Procedimiento de solución de un estudiante al reactivo 26. 
Con el propósito de resolver el problema del reactivo 26, el estudiante transforma las fracciones a decimales. Para ello, divide el numerador entre el denominador (como señalan las flechas) de cada fracción. Con las cantidades que obtiene realiza una suma $(0.66+0.16+0.08)$, cuyo resultado es 0.90 ; con base en tal cantidad intuye que, para alcanzar un entero, falta 0.10 que es igual $\frac{1}{10}$, por lo que selecciona la opción C. Con este procedimiento no es posible encontrar la respuesta correcta (opción D), pues el cociente de la división en la fracción $\frac{2}{3}$ es un número decimal periódico $(0.6666666 \overline{6})$ y el estudiante solo considera dos (0.66). Lo anterior llama la atención pues el reactivo consiste en un problema que puede ser resuelto obteniendo fracciones equivalentes. Esto es, calcular para $\frac{2}{3}, \frac{1}{6}$ y $\frac{1}{12}$ fracciones equivalentes que tengan denominador común, en este caso 12.

En el examen se incluyeron problemas en los que era necesario usar dos algoritmos (suma, resta, multiplicación o división) que involucraron fracciones para resolverlos. El porcentaje de respuesta correcta para estos reactivos aparece en la Tabla 8. Fue en este conjunto de reactivos donde se registró el porcentaje de respuesta correcta más bajo. Estos resultados coinciden con el estudio de Newton (2008), quien encontró en estudiantes para profesores un mayor número de errores en tareas de resolución de problemas de multiplicación y división de fracciones. Entre los datos mostrados resalta el reactivo 25, donde se planteó encontrar el área de un triángulo rectángulo, lo cual implicaba, primero, realizar una multiplicación y luego una división.

Tabla 8. Reactivos que implican el uso de dos operaciones básicas con fracciones.

\begin{tabular}{clc}
\hline Reactivo & \multicolumn{1}{c}{ Especificación } & $\begin{array}{c}\text { \% de respuesta } \\
\text { correcta }\end{array}$ \\
\hline 22 & $\begin{array}{l}\text { Resolver problemas que implican sumar y restar } \\
\text { fracciones propias. }\end{array}$ & 55.8 \\
25 & $\begin{array}{l}\text { Resolver problemas que implican multiplicar y dividir } \\
\text { fracciones propias. }\end{array}$ & 44.6 \\
& $\begin{array}{l}\text { Resolver problemas que implican multiplicar y restar } \\
\text { fracciones (una propia y otra impropia). }\end{array}$ & 61.0 \\
\hline
\end{tabular}

El reactivo 25 se muestra en la Figura 6 . Se esperaba que los estudiantes realizaran la multiplicación entre las fracciones $\frac{1}{2}$ y $\frac{4}{5}$; después una división del producto obtenido $\left(\frac{4}{10}\right)$ entre 2, para con ello obtener la respuesta correcta 
(opción C). Sin embargo, cerca de 40\% (120) de los futuros profesores eligieron la opción B; esta respuesta se obtiene al omitir la división. La mayor proporción de quienes respondieron de manera incorrecta fueron alumnos de la Normal rural (86), aunque en la Normal urbana una cantidad considerable (63 estudiantes) también respondió de forma equivocada. El error que cometen los estudiantes puede no deberse a su conocimiento acerca de cómo resolver algoritmos con fracciones, sino más bien a la omisión de algún "paso" (la división entre dos, por ejemplo) al emplear de manera inadecuada la fórmula para calcular el área de un triángulo, lo que, de todas formas, muestra una comprensión deficiente del problema planteado. Ser capaces de realizar los algoritmos pero sin tener una comprensión global del problema (que implica el reconocimiento de valores implícitos) parece reflejar un conocimiento del contenido matemático fragmentado, como lo refiere Newton (2008).

25. Calcule el área de la jardinera triangular que se muestra en seguida.
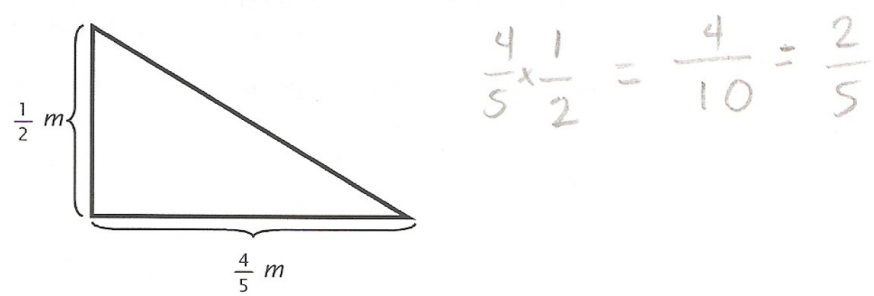
A) $\frac{13}{10} m^{2}$
B) $\frac{2}{5} m^{2}$
C) $\frac{1}{5} m^{2}$
D) $\frac{3}{10} m^{2}$

Figura 6. Procedimiento de solución de un estudiante al reactivo 25.

El reactivo 22 es otro de los que llama la atención por el bajo porcentaje de respuesta correcta. En él se pretendía que los futuros maestros realizaran una suma de fracciones que incluía tres sumandos, para luego restar el resultado a un total no explícito (véase Figura 7). 


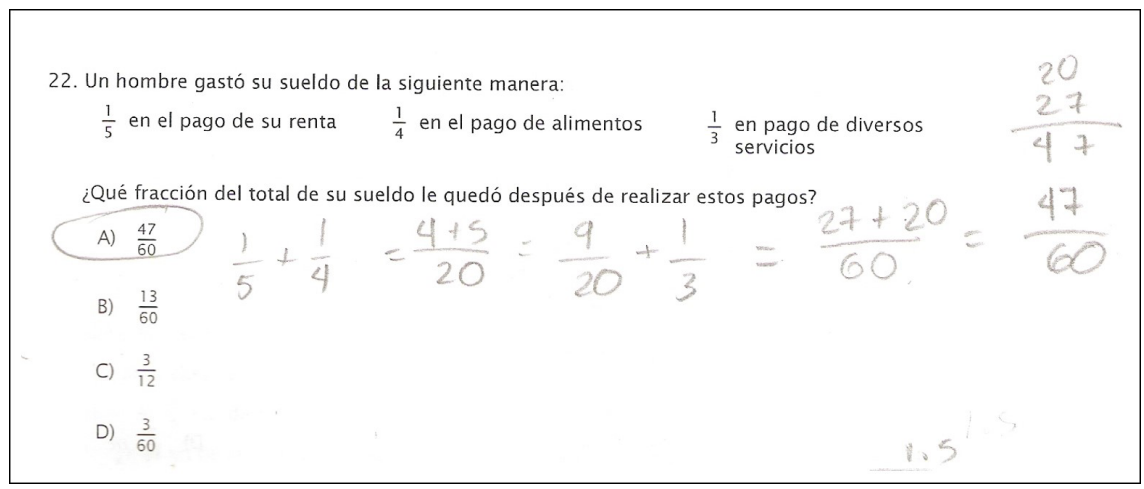

Figura 7. Procedimiento de solución de un estudiante al reactivo 22.

El proceso de solución de la Figura 7 muestra cómo cerca de una cuarta parte de los estudiantes (23\%) resolvió el problema. Se observa que el estudiante suma dos de las fracciones $\frac{1}{5}+\frac{1}{4}$; al resultado $\left(\frac{9}{20}\right)$ suma la otra fracción $\left(\frac{1}{3}\right)$. Con ello demuestra que tiene el conocimiento para resolver sumas de fracciones propias empleando el algoritmo convencional de la suma de fracciones. El resultado que obtiene $\left(\frac{47}{60}\right)$ no responde correctamente el problema, pues es necesario restar esta cantidad a un total no explícito, es decir, el sueldo del sujeto mencionado en el problema, y que para solucionarlo debe ser un entero. Este es uno de los problemas en el aprendizaje de las fracciones al que Fandiño (2009) Ilama "dificultades en la realización de operaciones" con fracciones; es uno de los temas que durante mucho tiempo ha sido de interés para los investigadores en el área de didáctica de las matemáticas. Fandiño (2009) señala que uno de los errores que con mayor frecuencia cometen los alumnos es identificar cuándo se deben emplear qué operaciones, tal y como sucede en el ejemplo de la Figura 7, en donde el participante solo usa un algoritmo (suma).

En el examen también se exploraron conocimientos acerca del manejo de algunas propiedades de las fracciones, como la equivalencia o la densidad. La Tabla 9 ofrece el porcentaje de aciertos para estos reactivos. En este conjunto de reactivos se incluyeron otras preguntas relacionadas con ubicar fracciones en la recta numérica, o el orden de estos números. Se puede decir que los estudiantes que participaron en el estudio son capaces de resolver problemas cuya solución implica el conocimiento de alguna de las propiedades de las fracciones que se preguntaron en el examen. 
Tabla 9. Reactivos que implican el manejo de propiedades de las fracciones.

\begin{tabular}{clc}
\hline Reactivo & \multicolumn{1}{c}{ Especificación } & $\begin{array}{c}\text { \% de respuesta } \\
\text { correcta }\end{array}$ \\
\hline 7 & $\begin{array}{l}\text { Resolver problemas que implican el uso de fracciones } \\
\text { equivalentes. }\end{array}$ & 72.9 \\
8 & $\begin{array}{l}\text { Resolver problemas que implican ubicar fracciones en la } \\
\text { recta numérica. }\end{array}$ & 72.9 \\
9 & $\begin{array}{l}\text { Resolver problemas que implican la propiedad de } \\
\text { densidad de los números fraccionarios. } \\
24\end{array}$ & $\begin{array}{l}\text { Resuelve problemas que implican ordenar números } \\
\text { fraccionarios. }\end{array}$ \\
\hline
\end{tabular}

Con el reactivo 9, que registró el porcentaje más bajo en este grupo problemas, se intentó explorar el conocimiento de los futuros profesores para encontrar una fracción contenida entre otras dos distintas: la densidad. La propiedad de densidad de una fracción significa que entre cualesquiera dos fracciones distintas puede siempre encontrarse otra fracción (Peterson y Hashisaki, 1980). En la Figura 8 se muestra el reactivo 9 del examen administrado. Al analizar las respuestas de los estudiantes resalta que 45 eligieron la opción A como correcta, aunque no lo fuera. Esta opción, $\frac{2}{3}$, pareciera ser la que responde al problema, pues tanto el numerador como el denominador, si se toman como números naturales, se encuentran entre el numerador y denominador de las fracciones $\frac{1}{2}$ y $\frac{3}{5}$. De nueva cuenta, la mayor cantidad de participantes que respondieron de forma incorrecta el reactivo se presentó en la Normal rural.

9. ¿Cuál de los siguientes números se encuentra entre $\frac{1}{2}$ y $\frac{3}{5}$ ?
A) $\frac{2}{3}$
B) $\frac{11}{10}$
C) $\frac{5}{7}$
D) $\frac{11}{20}$

Figura 8. Reactivo 9. Examen de conocimientos matemáticos. 
La Tabla 10 presenta los porcentajes de respuesta correcta para dos preguntas que involucraron la realización de operaciones básicas con números decimales y fracciones. El porcentaje más bajo se registró en el reactivo 18, en el cual se esperaba que los estudiantes emplearan una división (entero entre fracción mixta) para resolverlo.

Tabla 10. Reactivos que implican operaciones básicas con números decimales y fracciones.

\begin{tabular}{clc}
\hline Reactivo & \multicolumn{1}{c}{ Especificación } & $\begin{array}{c}\text { \% de respuesta } \\
\text { correcta }\end{array}$ \\
\hline 14 & $\begin{array}{l}\text { Resolver problemas que implican división de } \\
\text { fracciones entre números enteros. }\end{array}$ & 86.2 \\
18 & $\begin{array}{l}\text { Resolver problemas que implican dividir un } \\
\text { número entero entre una fracción mixta. }\end{array}$ & 74.7 \\
\hline
\end{tabular}

Al analizar las respuestas de los alumnos se observó que hubo quienes recurrieron a transformar la fracción a números decimales para hacer más fácil el manejo (ver Figura 9) y a un procedimiento de tanteos que, en este ejemplo, solo lo acercó a una de las opciones de respuesta. Como reporta Gairín (2004), en este caso la relación entre fracciones y decimales se establece mediante procesos algorítmicos, más que conceptuales. Con ello se confirma que a los estudiantes les resulta más sencillo el manejo de los números decimales pues, como mencionamos, se observó que emplearon esta estrategia para resolver otros reactivos. Fueron 51 estudiantes de la Normal rural quienes registraron el menor porcentaje de aciertos, en comparación con los 17 alumnos de la Normal urbana que respondieron incorrectamente este reactivo.

18. En la empresa "El formal" se confeccionan uniformes escolares; si para una falda talla 10 se utiliza $1 \frac{2}{5} \mathrm{~m}$ de tela, ¿cuántas faldas de la misma talla se confeccionarán con $35 \mathrm{~m}$ de tela?
A) 20
C) 26
D) 28
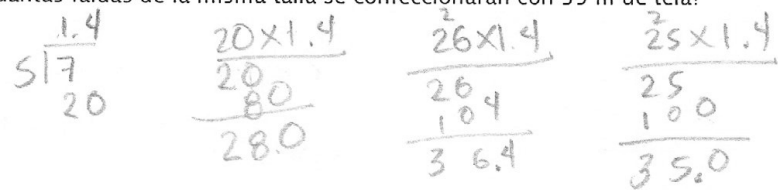

Figura 9. Proceso de solución de un estudiante al reactivo 18. 
Vemos, pues, que los resultados del examen proporcionan un panorama de cuáles son los conocimientos sobre fracciones y decimales de los aspirantes a profesores. Como señalamos, el porcentaje de respuesta correcta es más bajo en los reactivos que involucraron el uso de fracciones que aquellos que implicaron números decimales.

\section{CONCLUSIONES}

El artículo reporta el análisis del Conocimiento Común del Contenido Matemático sobre fracciones y decimales de los estudiantes para profesores de educación primaria. Lo anterior a través de las respuestas a un examen con reactivos que involucraron el uso de fracciones y decimales. Estamos convencidos de la importancia del conocimiento común de los contenidos matemáticos en el quehacer de los docentes. Nos interesamos por estudiantes para profesores porque estamos seguros de que es una etapa fundamental para la formación matemática.

Partimos de la idea de Ball, Thames y Phelps (2008) cuando mencionan que los maestros necesitan un conocimiento matemático que les permita resolver los problemas y ejercicios que se proponen en el nivel educativo donde trabajan. En el caso de los futuros profesores, demostraron poseer el conocimiento que les permite resolver problemas que involucran fracciones o decimales, si bien estos problemas estuvieron basados en contenidos estudiados en la escuela primaria. Y esta es nuestra primera acotación: los estudiantes para profesores que participaron en nuestro estudio logran resolver problemas con fracciones y decimales diseñados para alumnos que estudian la educación primaria, en otras palabras, saben cómo operar con este conjunto de números, por ejemplo, resolver problemas que implican algunas de las operaciones básicas. Aunque, como se observa en el análisis de la información, existen algunos estudiantes que no lo hacen, así como ciertos contenidos que, a pesar de ser muy elementales, no fueron resueltos correctamente por un porcentaje considerable de participantes.

Somos conscientes de que poseer un Conocimiento Común del Contenido no es suficiente para la enseñanza en matemáticas, éste se complementa con los otros tipos de conocimiento o subdominios. Consideramos que no basta con que un profesor pueda resolver problemas o ejercicios del grado o nivel educativo donde se desempeña, sino que es necesario que sea capaz de enfrentar problemas de grados o niveles educativos superiores. Estaríamos entonces, 
pisando el terreno del Conocimiento en el Horizonte Matemático y del Conocimiento Especializado del Contenido (Ball, 2003; Ball, Thames y Phelps, 2008).

El análisis de los resultados refleja que los aspirantes a profesores, en general, resuelven de mejor manera los reactivos que involucran números decimales, no así aquellos que implicaron el uso de fracciones. En el análisis de las formas en que resuelven los reactivos propuestos pudimos detectar que cuando intentan resolver problemas con fracciones, optan por transformarlas en números decimales, de tal manera que les resulte más fácil el tratamiento. Quizá sea porque una ventaja de los números decimales sobre las fracciones comunes es que resulta más fácil operar con ellos, pues los algoritmos son los mismos que se emplean en los números naturales, solo que existe dificultad al elegir el lugar en dónde se debe colocar el punto decimal en el resultado (Ávila y García, 2008). Sin embargo, como se mostró en los ejemplos presentados, esto no necesariamente conduce al resultado correcto.

Detectamos errores en los procedimientos de solución que son elementales en el manejo de fracciones o decimales. Por ejemplo, hubo participantes que, al resolver un problema que implicó suma de decimales, colocaron de forma incorrecta las cantidades (véase Figura 3). También se encontró que en algunos procedimientos de solución omitieron "un paso" para obtener la respuesta correcta (véase Figura 7). Más todavía, algunos resolvieron los problemas descartando opciones de respuesta (por eliminación), como se observa en la Figura 9.

Aunque los futuros profesores cuentan con un Conocimiento Común del Contenido sobre los temas que involucran decimales y fracciones en la educación primaria, nos dimos cuenta de que en una proporción considerable de ellos prevalecen errores que no se superaron en los niveles educativos previos y que los estudios superiores en la Escuela Normal no pudieron corregir. Como bien señalan Contreras et al. (2012), las instituciones formadoras de profesores deben asumir el reto de reforzar o incluso completar la formación matemática de los futuros maestros.

Encontramos diferencias entre el promedio de aciertos de los estudiantes de ambas normales. Quienes asistían a una Normal rural, en general, obtuvieron promedios más bajos que quienes pertenecían a una Normal urbana. Creemos que estas diferencias se deben a que los alumnos de las Normales rurales generalmente provienen de medios sociales más desfavorecidos y con antecedentes escolares menos favorables. No obstante estos resultados generales, llama la atención que algunos grupos de la Escuela rural tengan promedios similares a ciertos grupos de la Escuela urbana. El problema de la desigualdad 
no ocurre únicamente entre la ubicación de las escuelas (rural o urbana), también se aprecia al interior de cada una, debido tal vez a los procesos de selección y conformación de los grupos, o a los profesores asignados a cada grupo.

Consideramos que el principal aporte de este artículo es mostrar las fortalezas en el Conocimiento Común del Contenido matemático de futuros profesores en un tema que históricamente ha sido complejo, tanto para la enseñanza como para el aprendizaje. Aunque también pone de manifiesto las debilidades de los estudiantes, las que sin duda se llevarán hasta el trabajo docente, es decir, los profesores que no tienen un conocimiento consolidado finalmente compartirán sus errores con los alumnos a quienes enseñarán.

Pensamos que la enseñanza de temas que incluyen fracciones y decimales, o de cualquier otro objeto matemático exige, y lo subrayamos, un Conocimiento Común del Contenido consolidado. Además, consideramos que esta consolidación debe lograrse desde y en la formación inicial de los maestros. Es necesario que los futuros profesores (re)construyan los conocimientos acerca de este conjunto de números que adquirieron durante su paso por educación básica y media superior.

Por último, queremos resaltar que, si bien en este artículo solamente exploramos el subdominio del Conocimiento Común del Contenido, somos conscientes de que existe una serie de relaciones entre los diferentes dominios que conforman el MKT, las cuales exploraremos en un estudio futuro.

\section{REFERENCIAS}

Abad, F., García, C., Gil, B., Olea, J., Ponsoda, V. y Revuelta, J. (2004). Introducción a la Psicometría. Teoría Clásica de los Test y Teoría de la Respuesta al Ítem. Universidad Autónoma de Madrid. Recuperado de https://www.uam.es/personal_pdi/psicologia/ cadalso/Docencia/Psicometria/Apuntes/tema1TyP_4.pdf.

Ávila, A. y García, S. (2008). Los decimales más que una escritura. México, D.F.: Instituto Nacional para la Evaluación de la Educación.

Ball, D. (1990). Halves, Pieces, and Twoths: Constructing Representational Contexts in Teaching Fractions. Craft Paper 90-2. Recuperado de http://eric.ed.gov/?id=ED324226.

Ball, D. (2000). Bridging Practices. Intertwining Content and Pedagogy in Teaching and Learning to Teach. Journal of Teacher Education, 51(3), 241-247.

Ball, D. (2003). What Mathematical Knowledge is Needed for Teaching Mathematics. Secretary's Summit on Mathematics, US Department of Education. Recuperado de http:// www.erusd.k12.ca.us/projectalphaweb/index_files/MP/BallMathSummitFeb03.pdf. 
Ball, D. (2005). Knowing Mathematics for Teaching. American Educator, 1(1), 15-23.

Ball, D., Bass, H., Hill, H., Sleep, L., Phelps, G. y Thames, M. (2006). Mathematics Teaching and Learning to Teach. Presented at the Learning Network Conference Teacher Quality, Quantity and Diversity, Washington, DC.

Ball, D., Thames, M. y Phelps, G. (2008). Content Knowledge for Teaching What Makes It Special? Journal of Teacher Education, 59(5), 389-407. https://doi. org/10.1177/0022487108324554.

Carrillo, J., Climent, N., Contreras, L. C. y Muñoz-Catalán, M. (2013). Determining Specialized Knowledge for Mathematics Teaching. Proceedings of CERME 8. Recuperado de http://cerme8.metu.edu.tr/wgpapers/wg17_papers.html.

Centeno, J. (1988). Los números decimales. ¿Por qué?, ¿Para qué? Madrid, España: Síntesis.

Contreras, L., Carrillo, J., Zakaryan, D., Muñoz-Catalán, M. y Climent, N. (2012). An Exploratory Study about Student Teachers' Numerical Competences. Bolema: Boletim de Educação Matemática, 26(42B), 433-458. doi.org/10.1590/ S0103-636X2012000200003.

Durmuş, S. (2005). Identifying Pre-service Elementary School Teachers' Conceptualization Levels of Rational Numbers. Educational Sciences: Theory y Practice, 5(2), 659-665.

Fandiño, M. (2009). Las fracciones. Aspectos conceptuales y didácticos. Colombia: Magisterio.

Fernández, S. y Figueiras, L. (2010). El conocimiento del profesorado necesario para una educación matemática continua. En M. M. Moreno, A. C. Estrada, J. Carrillo y T. A. Sierra (eds.), Investigación en Educación Matemática (pp. 291-301). Lleida. Recuperado de http://funes.uniandes.edu.co/1696/.

Flores, G. y Díaz, M. (2016). Resultados Nacionales del Tercer Estudio Regional Comparativo y Explicativo. TERCE 2013. México: Instituto Nacional para la Evaluación de la Educación.

Flores-Medrano, E., Escudero, D. y Carrillo, J. (2013). A Theoretical Review of Specialized Content Knowledge. Proceedings of CERME 8. Recuperado de https://www.researchgate.net/publication/260266162.

Gairín, J. (2013). Estudiantes para maestros: reflexiones sobre la instrucción en los números racionales positivos. Contextos Educativos. Revista de Educación, 0(6), 235-260. doi.org/10.18172/con.538.

Godino, J., Batanero, C. y Font, V. (2007). Un enfoque ontosemiótico del conocimiento y la instrucción matemática. ZDM. The International Journal on Mathematics Education, 39 (1-2), 127-135. 
Hill, H., Schilling, S. y Ball, D. (2004). Developing Measure of Teachers Mathematics Knowledge for Teaching. The Elementary School Journal, 105. doi: 10.1086/428763.

Hill, H., Rowan, B. y Ball, D. (2005). Effects of Teachers' Mathematical Knowledge for Teaching on Student Achievement. American Educational Research Journal, 42(2), 371-406. doi.org/10.3102/00028312042002371.

Hill, H., Ball, D. y Schilling, S. (2008). Unpacking Pedagogical Content Knowledge: Conceptualizing and Measuring Teachers' Topic-Specific Knowledge of Students. Journal for Research in Mathematics Education, 39(4), 372-400.

Instituto Nacional para la Evaluación de la Educación. (2013). El aprendizaje en sexto de primaria en México, informe sobre los resultados Excale 06, aplicación 2009. Español, Matemáticas, Ciencias Naturales y Educación Cívica. México: INEE.

Instituto Nacional para la Evaluación de la Educación. (2015). Plan Nacional para la Evaluación de los aprendizajes 2015. Resultados Nacionales de Logro. Informe de resultados, México, D.F. Recuperado de http://planea.sep.gob.mx/content/ba/ docs/2015/estadisticas/Resultados_Nacionales_Logro.pdf.

Kieren, T. (1988). Personal Knowledge of Rational Numbers: Its Intuitive and Formal Development. In Number-concepts and Operations in the Middle Grades (pp. 53-92). Reston, VA: National Council of Teachers of Mathematics.

Martínez, M., Giné, C., Fernández, S., Figueiras, L. y Deulofeu, J. (2011). El conocimiento del horizonte matemático: más allá de conectar el presente con el pasado y el futuro. En M. Margarita, F. Gabriel, B. Lorenzo y P. María Mercedes (eds.), Investigación en Educación Matemática XV (pp. 429-438). Ciudad Real: Sociedad Española de Investigación en Educación Matemática. Recuperado de http://funes.uniandes.edu. co/1827/.

Montes, M., Contreras L., Liñán, M., Muñoz-Catalán, M., Climent, N. y Carrillo, J. (2015). Conocimiento de aritmética de futuros maestros: debilidades y fortalezas. Revista Educación, 367, 36-62. doi.org/10.4438/1988-592X-RE-2015-367-282.

Newton, K. (2008). An Extensive Analysis of Preservice Elementary Teachers' Knowledge of Fractions. American Educational Research Journal, 45(4), 1080-1110. doi. org/10.3102/0002831208320851.

Peterson, J. y Hashisaki, J. (1980). El sistema de los números racionales. En Teoría de la aritmética (pp. 197-245). México, D.F.: Limusa.

Pino-Fan, L. y Godino, J. (2015). Perspectiva ampliada del conocimiento didáctico-matemático del profesor. Paradigma, XXXVI(1), 87-109. Recuperado de http://docente. ulagos.cl/luispino/wp-content/uploads/2015/07/2662-6235-1-PB.pdf.

Schoenfeld, A. y Kilpatrick, J. (2008). Toward a Theory of Proficiency in Teaching Mathematics. En International Handbook of Mathematics Teacher Education: Vol. 2. Tools and 
Processes in Mathematics Teacher Education. Sense Publishers. Recuperado de http:// vocserve.berkeley.edu/faculty/AHSchoenfeld/Schoenfeld_Teaching_Proficiency.pdf.

Secretaría de Educación Pública. (2011). Plan de Estudios 2011. SEP.

Secretaría de Educación Pública. (2013). Aritmética, su aprendizaje y enseñanza (Primera edición). México, D.F.: Secretaría de Educación Pública.

Shulman, L. S. (1986). Those Who Understand: Knowledge Growth in Teaching. Educational Researcher, 15(2), 4-14. doi.org/10.3102/0013189X015002004.

Shulman, L. (1987). Knowlendge and Teaching Fundations of the New Reform. Harvard Educational Review, 52(1). Recuperado de http://people.ucsc.edu/ ktellez/shulman.pdf. Sosa, L. (2011). Conocimiento matemático para la enseñanza en bachillerato: un estudio de dos casos. (Tesis de doctorado). Universidad de Huelva, Huelva, España. 